\title{
KIC 2831097
}

\section{A short orbital-period candidate RR Lyrae binary}

\author{
Ádám Sódor ${ }^{1, \star}$, Marek Skarka ${ }^{1, \star \star}$, Jiří Liška ${ }^{2,3, \star \star \star}$, and Zsófia Bognár ${ }^{1, \star \star \star \star}$ \\ ${ }^{1}$ Konkoly Observatory, MTA Research Centre for Astronomy and Earth Sciences, Konkoly Thege út 15-17, \\ H-1121 Budapest, Hungary \\ ${ }^{2}$ Department of Theoretical Physics and Astrophysics, Masaryk University, Kotlářská 2, CZ-611 37 Brno, \\ Czech Republic \\ ${ }^{3}$ CEITEC BUT, Purkyn̆ova 656/123, CZ-612 00 Brno, Czech Republic
}

\begin{abstract}
KIC 2831097 was discovered to be a first-overtone RR Lyrae pulsator based on 4-year Kepler photometry (Sódor et al. 2017, MNRAS, 465, L1). The data show strong, $0.1 \mathrm{~d}$ amplitude systematic phase variations that can be explained by light travel-time effect caused by an about 2-year period orbital motion in a binary system, superimposed on a linear pulsation-period decrease. To verify the binary hypothesis, several well-timed radial-velocity observations will be sufficient.
\end{abstract}

\section{Introduction}

KIC 2831097 is the fifth first-overtone Kepler ([2]) RR Lyrae (RRc) star with detailed analysis published (first four: [3]; KIC 2831097: [1]). The pulsation properties of the star were found to be typical compared with the four previously known Kepler RRc stars (see details in [1]).

The $O-C$ variations are shown in Figure 1. We modelled the $O-C$ data by the superposition of a constant period chane and the orbital light-travel-time effect ([1]).

\section{Verification of the binarity}

Even though almost two hypothetical orbital revolutions appear in the $O-C$ data, the intrinsic origin of these phase variations cannot be excluded (see details and discussion in [1]). Therefore, radialvelocity (RV) measurements are needed in order to confirm or reject the binarity. Since the hypothetical orbit is short and the radial-velocity amplitude is large, the verification can be attained in two consecutive observing seasons with high confidence, according to Figure 2.

The organization of the observations are underway. Meanwhile, we are also collecting new $O-C$ data to extend the Kepler baseline.

\footnotetext{
${ }^{\star}$ sodor@konkoly.hu

$\star \star$ marek.skarka@csfk.mta.hu

$\star \star \star$ jiriliska@post.cz

$\star \star \star \star$ bognar@konkoly.hu
} 

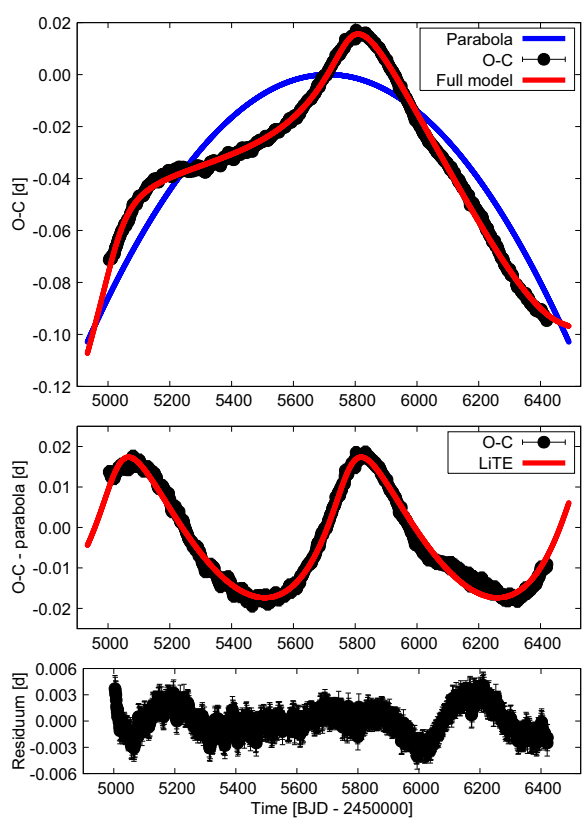

Figure 1. $O-C$ data of KIC 2831097, and the constant period change (blue) and orbital light-travel-time (red) models.

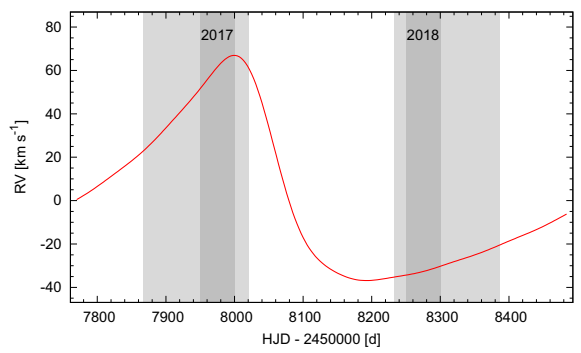

Figure 2. Predicted RV curve of KIC 2831097 for the 2017-2018 observing seasons, based on the orbital solution.

Acknowledgments: The financial support of the Hungarian NKFIH Grants K-115709, K-113117 and K-119517 are acknowledged. ÁS was supported by the János Bolyai Research Scholarship of the Hungarian Academy of Sciences. MS acknowledges the support of the postdoctoral fellowship programme of the Hungarian Academy of Sciences at the Konkoly Observatory as a host institution.

\section{References}

[1] Sódor, Á., Skarka, M., Liška, J., \& Bognár, Zs., MNRAS, 465, L1 (2017)

[2] Borucki, W. J., Koch, D., \& Basri, G., Science, 327, 977 (2010)

[3] Moskali P., Smolec, R., \& Kolenberg, K., MNRAS, 447, 2348 (2015) 